\title{
On the resolution of critical flow regions in inviscid linear and nonlinear instability calculations
}

\author{
VASSILIOS THEOFILIS \\ DLR, Institute for Fluid Mechanics, Bunsenstraße 10, D-37073 Göttingen, Germany
}

\begin{abstract}
Numerical methods for tackling the inviscid instability problem are discussed. Convergence is demonstrated to be a necessary, but not a sufficient condition for accuracy. Inviscid flow physics set requirements regarding grid-point distribution in order for physically accurate results to be obtained. These requirements are relevant to the viscous problem also and are shown to be related to the resolution of the critical layers.

In this respect, high-resolution nonlinear calculations based on the inviscid initial-boundary-value problem are presented for a model shear-layer flow, aiming at identification of the regions that require attention in the course of high-Reynolds-number viscous calculations. The results bear a remarkable resemblance with those pertinent to viscous flow, with a cascade of high-shear regions being shed towards the vortex-core centre as time progresses. In parallel, numerical instability related to the finite-time singularity of the nonlinear equations solved globally contaminates and eventually destroys the simulations, irrespective of resolution.
\end{abstract}

Keywords: direct numerical simulation, transition to turbulence, Euler equations, finite-time singularities.

\section{Introduction, physical background and motivation}

One of the aspects characterising laminar-turbulent flow transition is the generation of smallscale structures as the flow progresses from laminar through transitional into a turbulent state. As a matter of fact, the scales most difficult to be resolved accurately are those generated during transition. In the context of numerical calculations of any of the transition stages this translates into the requirement to employ numerical methods of highest accuracy and lowest dissipation possible. Spectral or finite-difference type methods of high formal accuracy are routinely used to calculate linear growth, nonlinear saturation in two-dimensions, and breakdown stages in three-dimensional viscous wall-bounded and free-shear flows [1].

Inviscid linear theory, on the other hand, keeps forming an integral part of current research into the mechanisms responsible for transition, especially relevant to compressible (in particular supersonic) flows, where inviscid instabilities are often prevalent (Mack [2], Duck [3]). The topic in its physical aspects is current research and too wide to be introduced exhaustively. The interested reader may find elements of the early inviscid linear theory in Lin [4], more recent successes are summarised by Mack [2] and new instability modes are constantly being discovered (Macaraeg and Streett [6], Shaw and Duck [7]). In parallel, a number of investigators are actively pursuing the nonlinear aspects of inviscid theory (Smith and Burggraf [8], Kachanov et al. [9], Ryzhov [10]). In the latter context, analysis is based on nonlinear systems of equations of, typically, the Benjamin-Ono class which are treated by combined analytical and numerical methods. From a physical point of view the novel aspect introduced by addressing the inviscid system of equations is, of course, the admittance in the latter of discontinuous solutions. 
Two requirements emerge for the numerical calculations; on the one hand, the solutions to be obtained should be as accurate as possible, given a maximum affordable discretisation, on the other hand, the numerical method adopted should be able to treat discontinuities. The second requirement may lead to the use of finite-difference-type methods which, owing to their inherent dissipation, can either resolve potentially existing discontinuities or localise the effect of their poor resolution. However, the amount of dissipation present in a finite-difference method is inversely proportional to the formal accuracy of the method, suggesting the need for finite-difference methods of high formal order. Even then, the low scales generated during the late transitional stages, corresponding to the high wave numbers which are damped in a finite-difference scheme, may be poorly represented. Spectral methods, on the other hand, are known to be optimally suited for the problem of linear and nonlinear instability and transition, where a reasonable resolution is to be devoted to extraction of as much physically relevant information as possible. However, the global expansion character of spectral methods suggests that the existence of a true discontinuity at some region in the flow will generate numerical instability which will contaminate the flow field globally; it appears that the requirements of optimal accuracy and resolution of true discontinuities are contradictory. A distinction should be made here between true discontinuities, like a shock, and solitary-wave type of solutions, such as those arising in the numerical solution to Burgers, Korteweg-de-Vries, or BenjaminOno equations. In practice, it is the latter type of steep gradient which appears in the nonlinear stages of transition simulations. A brief discussion of this issue of resolution of regions where steep, yet finite gradients appear in the flow may be found in the Appendix.

There is a further issue involved in transition modelling which seems to have received attention in its own right only in the context of compressible simulations [11], although relevant in both incompressible and compressible flow. This is related to the distribution of the limited number of points which one has available in the course of a simulation. Numerical experience suggests that points be placed such that they resolve well the (known in advance) regions of high gradients in the basic flow. In the fortuitous case that these regions coincide with the critical layers predicted by linear theory the delivered nonlinear simulation results can be reliable. Otherwise, solutions may be obtained which bear no resemblance to the physical problem, even though purely numerical criteria suggest that they have converged. Critical layers develop early in the process of transition, during the stage of linear growth of perturbations. Inviscid linear analysis suggests that their neighbourhood is the flow region requiring optimal resolution, since linear perturbations in that flow region typically possess very steep (though finite) gradients. If credibility is to be given to the nonlinear equilibria in two spatial dimensions (Koch, [12]) or transitional flow fields in three spatial dimensions which follow linear growth, linear critical layers must be well resolved. If a low-order accurate numerical method is used for the simulation, the error introduced by the inaccurate description of the physics of the critical layer region may go unnoticed or be confused with other sources of numerical error; the accuracy of the nonlinear equilibria resulting from such simulations is questionable. This is the reason why we adhere to a fully spectral method, the accuracy properties of which permit clear manifestation of the singularities developing.

The present paper is firstly concerned with the issue of the significance of critical-layer resolution by reference to an analytically known example. The conclusions drawn in a linear framework carry the same weight in nonlinear calculations. In the latter case, though, comparisons of analytical and numerical solutions may not be as straightforward as is the case with classic inviscid linear-analysis results (Fjørtøft [13]). One has to rely on accurate numerical solutions, which can provide useful insight for further nonlinear analysis. 
The main part of the present paper is a contribution in this direction. We perform direct numerical simulations of the compressible Euler equations, motivated by an issue which was raised during three-dimensional viscous and inviscid shear-layer transition simulations, namely the appearance in the late transitional stages of well-defined clusters of vorticity between the high-shear regions which outline the so-called braids. Since the two-dimensional model also exhibits such structures, we decided to probe the nonlinear regime of compressible model shear-layer inviscid instability, by numerical solution of the two-dimensional Euler equations at high resolution. Numerical aspects alone of the results obtained are discussed herein; the physical information is currently under evaluation. In this respect, no attempt will be made presently to discuss the physical aspects of the results obtained within the frame of finite-time singularities developing in the solution of the Euler equations [14]; such a discussion will follow the physical evaluation of our results and will be presented in due course.

The paper is structured as follows. The alternative forms of the governing equations solved are presented in Section 2. After exposing the link between the full equations and the linear problem solved in order to initialize the computations, we present in Section 3 the tools used for the direct Euler simulations. Particular reference is made to the collocation approach which is vastly inferior to the commonly used pseudospectral scheme on scalar machines, but turns out to be more efficient than non-optimised fast Fourier transforms on the vector machine used. The members of the Padé and Runge-Kutta families used, respectively, for evaluation of spatial derivatives and time-integration are identified. Accuracy and efficiency aspects of the nonlinear results obtained are treated in Section 4. After a brief discussion in a linear framework of the issue of resolution of the critical layer in Section 5 we present the results of the nonlinear simulations of inviscid instability in the compressible shear-layer flow. The overall picture obtained is discussed in the closing section.

\section{Governing equations}

\subsection{DIRECT NUMERICAL SIMULATIONS OF THE COMPRESSIBLE EULER EQUATIONS}

The compressible two-dimensional Euler equations may be used to describe inviscid flow disturbances of arbitrary magnitude. Time- and space-accurate numerical solutions of these equations, termed Direct Euler Simulations (DES), have been obtained. We have used both the conservative unsplit form of the equations (i.e. one in which flow quantities are not decomposed into basic and disturbance terms)

$$
\frac{\partial}{\partial t}\left(\begin{array}{c}
\rho \\
\rho u \\
\rho v \\
E
\end{array}\right)=-\frac{\partial}{\partial x}\left(\begin{array}{c}
\rho u \\
p+\rho u^{2} \\
\rho u v \\
u(E+p)
\end{array}\right)-\frac{\partial}{\partial y}\left(\begin{array}{c}
\rho v \\
\rho u v \\
p+\rho v^{2} \\
v(E+p)
\end{array}\right),
$$

where $E=\frac{1}{\gamma-1} p+\frac{1}{2} \rho\left(u^{2}+v^{2}\right)$ and $\gamma M^{2} p=\rho T$, as well as the non-conservative unsplit form of system (1). Here $M$ is the Mach-number and $\gamma$ the ratio of specific heats. Instability results obtained using the two forms were identical. Further, considering the decomposition 
into basic $\overline{\mathbf{q}}=(\bar{\rho}, \bar{u}, 0, \bar{p})^{\mathrm{T}}$ and disturbance $\hat{\mathbf{q}}=(\hat{\rho}, \hat{u}, \hat{v}, \hat{p})^{\mathrm{T}}$ flow quantities and substituting $\mathbf{q}=\overline{\mathbf{q}}+\hat{\mathbf{q}}$ in the system (1), we solved the following system of disturbance equations

$$
\begin{aligned}
& \frac{\partial \hat{\rho}}{\partial t}=-\left[\bar{u} \frac{\partial \hat{\rho}}{\partial x}+\bar{\rho} \frac{\partial \hat{u}}{\partial x}+\hat{u} \frac{\partial \hat{\rho}}{\partial x}+\hat{\rho} \frac{\partial \hat{u}}{\partial x}+\frac{d \bar{\rho}}{d y} \hat{v}+\bar{\rho} \frac{\partial \hat{v}}{\partial y}+\hat{v} \frac{\partial \hat{\rho}}{\partial y}+\hat{\rho} \frac{\partial \hat{v}}{\partial y}\right], \\
& \frac{\partial \hat{u}}{\partial t}=-\frac{1}{(\bar{\rho}+\hat{\rho})} \\
& {\left[\frac{\partial \hat{p}}{\partial x}+\overline{\rho u} \frac{\partial \hat{u}}{\partial x}+\bar{\rho} \hat{u} \frac{\partial \hat{u}}{\partial x}+\bar{u} \hat{\rho} \frac{\partial \hat{u}}{\partial x}+\hat{\rho} \hat{u} \frac{\partial \hat{u}}{\partial x}+\bar{\rho} \frac{d \bar{u}}{d y} \hat{v}+\bar{\rho} \hat{v} \frac{\partial \hat{u}}{\partial y}+\frac{d \bar{u}}{d y} \hat{\rho} \hat{v}+\hat{\rho} \hat{v} \frac{\partial \hat{u}}{\partial y}\right],} \\
& \frac{\partial \hat{v}}{\partial t}=-\frac{1}{(\bar{\rho}+\hat{\rho})}\left[\frac{\partial \hat{p}}{\partial y}+\bar{\rho} \bar{u} \frac{\partial \hat{v}}{\partial x}+\bar{\rho} \hat{u} \frac{\partial \hat{v}}{\partial x}+\bar{u} \hat{\rho} \frac{\partial \hat{v}}{\partial x}+\hat{\rho} \hat{u} \frac{\partial \hat{v}}{\partial x}+\bar{\rho} \hat{v} \frac{\partial \hat{v}}{\partial y}+\hat{\rho} \hat{v} \frac{\partial \hat{v}}{\partial y}\right], \\
& \frac{\partial \hat{p}}{\partial t}=-\left[\bar{u} \frac{\partial \hat{p}}{\partial x}+\hat{u} \frac{\partial \hat{p}}{\partial x}+\hat{v} \frac{\partial \hat{p}}{\partial y}+\frac{1}{M^{2}} \frac{\partial \hat{u}}{\partial x}+\frac{1}{M^{2}} \frac{\partial \hat{v}}{\partial y}+\gamma \hat{p} \frac{\partial \hat{u}}{\partial x}+\gamma \hat{p} \frac{\partial \hat{v}}{\partial y}\right] .
\end{aligned}
$$

\subsection{INVISCID LINEAR INSTABILITY OF PLANAR COMPRESSIBLE SHEAR FLOWS}

If we take $\hat{\mathbf{q}}=A \tilde{\mathbf{q}}$ with $A$ a constant amplitude parameter of small magnitude, then linearisation of the system (2-5) is permissible; the $O(A)$ disturbance equations which result read

$$
\begin{aligned}
& \frac{\partial \tilde{\rho}}{\partial t}=-\left[\bar{u} \frac{\partial \tilde{\rho}}{\partial x}+\bar{\rho} \frac{\partial \tilde{u}}{\partial x}+\frac{d \bar{\rho}}{d y} \tilde{v}+\bar{\rho} \frac{\partial \tilde{v}}{\partial y}\right], \\
& \frac{\partial \tilde{u}}{\partial t}=-\frac{1}{\bar{\rho}}\left[\frac{\partial \tilde{p}}{\partial x}+\overline{\rho u} \frac{\partial \tilde{u}}{\partial x}+\bar{\rho} \frac{d \bar{u}}{d y} \tilde{v}\right], \\
& \frac{\partial \tilde{v}}{\partial t}=-\frac{1}{\bar{\rho}}\left[\frac{\partial \tilde{p}}{\partial y}+\overline{\rho u} \frac{\partial \tilde{v}}{\partial x}\right], \\
& \frac{\partial \tilde{p}}{\partial t}=-\left[\bar{u} \frac{\partial \tilde{p}}{\partial x}+\frac{1}{M^{2}} \frac{\partial \tilde{u}}{\partial x}+\frac{1}{M^{2}} \frac{\partial \tilde{v}}{\partial y}\right] .
\end{aligned}
$$

At this point we may introduce an eigenmode ansatz on time into the system (6-9) and solve the resulting two-dimensional inviscid eigenvalue problem ( $c f$. Hall and Horseman [17]); such an approach is beyond the scope of the present investigation. Instead, the standard linear stability ansatz of streamwise periodicity of the flow considered is made, and all flow quantities are decomposed into their Fourier components in that direction; if we take a periodicity length $L_{x}$, a (real) streamwise wavenumber is defined as $\alpha=2 \pi / L_{x}$. The normal direction $y$, on the other hand, is taken to be unbounded and resolved. The disturbance quantities $\tilde{\mathbf{q}}$ then take the form $\tilde{\mathbf{q}}=\check{\mathbf{q}}(y) \mathrm{e}^{i(\alpha x-\Omega t)}+c . c$. with $\Omega=\alpha c$ and $\check{\mathbf{q}}$ being complex quantities and c.c. denoting complex conjugation in order for the physical space quantities $\tilde{\mathbf{q}}$ to remain real. Numerical solution of the inviscid compressible linear eigenvalue problem which results from substitution of the above ansatz in (6-9) delivers the eigenvalue $\Omega$ and eigenvector $\check{\mathbf{q}}$ 
pertinent to a given set of parameters $(\alpha, M)$. If $\mathfrak{F}\{\Omega\}>0$, exponential growth of $\breve{\mathbf{q}}$ (within the linear framework) will result. Such linearly unstable waves have been selected as initial conditions for the nonlinear calculations based on (1) or (2-5). The point(s) in the flow where the phase velocity of the unstable disturbances equals that of the basic flow play a central role in the analysis of inviscid instability results [4]; as such, their neighbourhood places additional demands on resolution.

\section{Numerical algorithms}

Both time and space have to be resolved for the nonlinear calculations based on either (1) or (2-5). The numerical methods used comprise standard technology of compressible direct numerical simulations. Accuracy and efficiency, in that order, are the main considerations in the long-time integrations of the equations which have to be performed. The numerical methods utilised follow from these two requirements, as well as the assumptions made on the flow problem considered.

The periodicity assumed in the streamwise direction leads to a Fourier expansion in this coordinate. Implicit in this decision is the inability to resolve discontinuities, such as shocks potentially present in the simulations as the Mach number increases (Vreman et al. [18]). No evidence of shocks exists, however, for this flow problem at the low Mach number at which the calculations were performed. In the normal direction an unbounded domain has to be treated and this offers some flexibility. Both finite-difference and spectral methods may, in principle, be used. The heavy work load associated with the dense matrices resulting from a spectral discretisation and the time-step limitation associated with the CFL condition in explicit time-integrations and, not least, hardware considerations led investigators in the late 80's to exploit finite-difference type of methods for transition simulations. We discuss comparisons between Chebyshev collocation, Chebyshev pseudospectral and Padé 3/4/6 (Lele [19], Adams [20]) discretisation with respect to accuracy and efficiency. In contrast to the aforementioned simulations, the results obtained on the vector machine led us to adhere to a fully spectral scheme.

As to the spectral discretization, essential references for spectral methods are Canuto et al. [31] and Boyd [33]. A step-by-step discussion of the relationship between the classic spectral space expansions and spectral collocation may be found in $\mathrm{Ku}$ and Hatziavramidis [21]. Grids and the pertinent collocation derivative matrices must be defined at the beginning of the calculations. We obtained results using two mappings between the Gauss-Lobatto points $x$ and the mapped coordinate $\eta \in(-\infty, \infty)$, one algebraic and one exponential. For eigenproblem calculations we employ spectral collocation in physical space, rather than the more traditional (and numerically equivalent) transform-space approach. For exploratory runs at low resolution $\left(<128^{2}\right)$ in the DES, collocation in physical space is also the method of choice; for high resolution runs on scalar machines transform methods are indispensable.

Efficiency is central to the success of long-time integrations and any candidate to match the accuracy properties of a spectral expansion must be considered. We have chosen to compare Chebyshev collocation against a Padé scheme which is 6th-order accurate in the interior of the domain, while a third- and fourth-order accurate closure is used in the boundary and immediately next to the boundary points. This scheme was proposed by Lele [19] and has been used by Sandham and Reynolds [22] in viscous compressible calculations of shear-layer 
flow transition and Adams [20] in transition simulation of compressible boundary-layer flow. Calculation of spatial derivatives is performed here by inversion of the matrix equation

$$
\mathbf{L} f^{\prime}=\frac{1}{\delta} \mathbf{R} f
$$

for the first derivative $f^{\prime}$ of a function $f$ defined on a domain which has been partitioned equidistantly with spacing $\delta$. Lele [19] gives a full discussion regarding prescriptions for higher derivatives, necessary in the course of viscous simulations. Numerically this scheme is attractive on two counts. First, the bandedness of the matrices $\mathbf{L}$ and $\mathbf{R}$ suggests that efficient linear-algebra methods may be used for the inversion of (10). Much more significantly, though, both dispersive and dissipative (the latter associated with the boundary treatment) errors of Padé schemes, are significantly lower than those of classic second- and fourth-order accurate finite-difference schemes [19], although higher than those of a spectral method. The scheme in discussion, thus, comes much closer to the resolving properties of spectral spectral methods than the classic finite-difference schemes. Given the quantum leaps in hardware technology over the decade that elapsed since the Padé schemes were proposed, it is of interest to examine whether a straightforward spectral discretisation is competitive in terms of efficiency; we address this issue in the following section.

Out of the plethora of alternatives discussed in the literature we have chosen one of the third-order explicit Runge-Kutta schemes proposed by Wray [23]

$$
\begin{array}{rl}
k_{1}=\Delta t & F\left(U^{n}, t_{n}\right) \\
k_{2}=\Delta t & F\left(U^{n}+\frac{2}{3} k_{1}, t_{n}+\frac{2}{3} \delta t\right) \\
k_{3}=\Delta t \quad F\left(U^{n}+\frac{1}{4} k_{1}+\frac{5}{12} k_{2}, t_{n}+\frac{2}{3} \delta t\right) \\
U^{n+1}=U^{n}+\frac{1}{4} k_{1}+\frac{3}{20} k_{2}+\frac{3}{5} k_{3},
\end{array}
$$

with index $n$ indicating time-level, $\Delta t$ being the time-step, $U$ the solution vector and $F$ the RHS vectors in (1) or (2-5). The attractive feature of the particular RK3 scheme is that it provides third-order accuracy in time, while it requires only two levels of storage. As such the RK 3 represents a compromise between the more accurate but more expensive classic RK4 and the less accurate and equally expensive second-order accurate RK2 scheme.

\section{Numerical aspects of the results}

\subsection{EFFICIENCY}

By far the most time-consuming element in direct numerical simulations is the calculation of the spatial derivatives in the right-hand sides of (1) or (2-5). Efficiency is of importance and it turns out that the optimal approach depends not only on the algorithm, but also on the machine architecture used. Table 1 shows the timing of the alternative approaches utilised presently.

These results deserve discussion with reference to the machine used. Albeit reasonably fast, the scalar machine is a typical example of the textbook application of transform algorithms, with the methods based on the FFTs resulting in dramatic savings compared with matrix multiplication for all but runs at exploratory resolution. When comparing timings between the fully 
Table 1. Times per time-step in seconds for the different approaches used for the calculation of spatial derivatives. In brackets the Mflops performed by the code on the vector machine.

\begin{tabular}{|c|c|c|c|c|c|c|}
\hline & SUN Ultra 1 & & & NEC SX-4 & & \\
\hline Resolution & Collocation & Pseudospectral & Padé & Collocation & Pseudospectral & Padé \\
\hline $32 \times 32$ & 0.809 & $0 \cdot 313$ & 0.257 & $0.005(163)$ & $0.075(21)$ & $0.040(21)$ \\
\hline $64 \times 64$ & 6.029 & $1 \cdot 242$ & 1.010 & $0.025(417)$ & $0.185(36)$ & $0.111(35)$ \\
\hline $128 \times 128$ & $46 \cdot 430$ & $5 \cdot 153$ & $4 \cdot 343$ & $0 \cdot 135(720)$ & $0.565(55)$ & $0 \cdot 350(51)$ \\
\hline $256 \times 256$ & 433.571 & 21.445 & 18.074 & $0.770(1046)$ & $1.725(79)$ & $1.130(69)$ \\
\hline $512 \times 512$ & $4382 \cdot 610$ & $86 \cdot 645$ & 73.084 & $4 \cdot 175(1545)$ & $5 \cdot 205(103)$ & $4.045(90)$ \\
\hline
\end{tabular}

spectral and the mixed spectral/finite-difference methods, on the other hand, we see that the latter is marginally more efficient than the former at the same resolution. Given the common use of a pseudospectral Fourier expansion in $x$ in both algorithms, the most probable explanation for this somewhat surprising result is the optimisation of the fast-Fourier transform library on the scalar machine. It is, however, erroneous to conclude from the results of Table 1 that the fully spectral scheme should be dropped in favour of the mixed spectral/finite-difference algorithm on grounds of efficiency. Regarding accuracy, we will demonstrate shortly, by reference to the linear stability growth rate reproduction by the nonlinear code, that the number of finite-difference points necessary to match the accuracy of the spectral expansion in $y$ is larger by an amount which has the potential to offset the benefit of the efficiency demonstrated here.

The results on the vector machine should serve as a warning against uncritical use of library software on a high-performance computer. While an increase of the workload associated with matrix multiplication in the collocation algorithm results in a better performance of the code, the latter reaching an acceptable 1.5 Gflops without optimisation, the one-dimensional FFT libraries utilised for the pseudospectral calculations and the relatively short vector-length tridiagonal matrix inversions in the Padé algorithm result in very poor performance. As a consequence, the direct algorithm based on collocation, which is inefficient on scalar machines, becomes very competitive on the supercomputer.

\subsection{ACCURACY}

As an initial condition for the nonlinear computations we impose the unstable wave delivered by linear theory at the Mach- and wave number parameters chosen for the nonlinear computations. The usual quality test, namely reproduction of the linear growth rate by the simulation code, is performed here under somewhat different conditions than those of a viscous temporal simulation. Specifically, in the present inviscid calculations there is no possibility to use a forcing term, as done in viscous simulations, in order to maintain the imposed basic flow profile a solution of the nonlinear system of equations; this would only be possible by including a vertical velocity component, which would imply a spatial simulation. Consequently, the growth rate obtained in the simulations decreases monotonically with time and comparisons with the linear growth rate are only possible at very early times in the simulation, provided that the amplitude at which the linear wave is superimposed is taken to be small and the time- 
Table 2. Comparisons of linear growth rates obtained by the Chebyshev pseudospectral and the Padé finite-difference differentiation algorithms in $y$; in both cases a Fourier expansion has been used in $x$. Also cited is the linear stability theory (LST) result, obtained by Chebyshev collocation. Parameters used: $M=0.4, \alpha=0.409$.

\begin{tabular}{llll}
\hline \multicolumn{3}{c}{ LST } & \multicolumn{2}{c}{ DES } \\
\hline Ny & $\Omega_{i}$ & $\Omega_{i}$ (Spectral) & $\Omega_{i}$ (Padé) \\
\hline 32 & 0.155332 & 0.156028 & 0.408829 \\
64 & 0.155301 & 0.155277 & 0.147440 \\
128 & 0.155301 & 0.155302 & 0.155195 \\
256 & 0.155301 & 0.155301 & 0.155298 \\
512 & 0.155301 & 0.155301 & 0.155302 \\
\hline
\end{tabular}

step much lower than the CFL-delivered time-step. This has been done for both schemes used to calculate spatial derivatives in $y$ and the results may be found in Table 2 .

We find both schemes capable of reproducing the linear-theory result, albeit that for results of the same level of accuracy Padé differentiation is inferior to a Chebyshev expansion for two reasons. First, at low resolution the spectral scheme delivers reasonable approximations of the physical result, while the finite-difference calculation has an order-of-magnitude error. Second, the number of points necessary for the finite-difference result to match in quality that of the spectral discretisation can be substantial. In view of this result, we refrained from the approach taken in earlier simulations [24], where we interchanged the two differentiation algorithms in calculating the $y$-derivatives in (1), always remaining within modest resolutions [24]. For the current nonlinear computations we solved (2-5), adhering to the fully spectral scheme.

\section{Linear and nonlinear instability results}

The issue which gave rise to the present nonlinear stability results is whether the structures that form between the high-shear regions during the late stages of compressible shear-layer transition are of physical origin. Such structures can be clearly seen in the results of Sandham and Reynolds [22] ( $c f$. in Figures 3.5b, 4.4b, 4.10c, 4.11c), while we encountered them also in the course of validation runs for direct simulations of compressible attachment-line instability [24]. The structures were, surprisingly, present also in inviscid calculations, irrespective of whether a two- or three-dimensional computation was performed, as can also be seen in Figure 2(a) in [24]. Although certainly not pointwise oscillations, their association with the tails of the vorticity contours gave rise to the suspicion that they might be due to numerical instability; however, if physical, their appearance already in the two-dimensional inviscid model, in conjunction with the regular pattern that they form, could have profound implications for theoretical models of the late stages of transition in viscous shear flows. 
However, before entering the discussing of the nonlinear stability results obtained, we briefly take a detour and expose the issue of resolution of the critical layer, in a linear framework and for a problem for which analytical results to compare against are well established.

\subsection{ON THE RESOLUTION OF LINEAR CRITICAL LAYERS}

The incompressible viscous linear stability of the asymptotic suction boundary-layer profile, whose basic flow is given by

$$
\bar{u}(y)=1-\mathrm{e}^{-y},
$$

is governed by the system derived and solved by Hocking [27]. As is the case with the generalised Hiemenz boundary layer [28, 29], the basic flow is an exact solution of the full Navier-Stokes equations of motion. Consequently, an inviscid stability analysis of this profile should not be expected to deliver physically interesting information at finite Reynolds numbers. However, Hughes and Reid [30] found an analytic solution of the Rayleigh equation pertinent to this profile. This may serve as an illustration of the difference between numerically converged and physically relevant solutions. Demanding decay at infinity and denoting the normal perturbation velocity eigenvector by $\Phi$ and the position of the critical layer by $y_{\mathrm{cr}}$, we have the solution

$$
\begin{aligned}
& \qquad \Phi(y)=\frac{F(p, q ; r ; t)}{F(p, q ; r ; 1)} \mathrm{e}^{-\alpha\left(y-y_{\mathrm{cr}}\right)} \\
& \text { for }|t|=\left|\mathrm{e}^{-\left(y-y_{\mathrm{cr}}\right)}\right| \leqslant 1 \text {, where } \\
& \quad p=\alpha+\sqrt{\left(1+a^{2}\right) ; \quad q=\alpha-\sqrt{(}\left(1+a^{2}\right) ; \quad r=1+2 \alpha}
\end{aligned}
$$

and $F(p, q ; r ; t)$ is the standard hypergeometric function. Hughes and Reid [30] also provided the asymptotic limit of Equation (12) as $y \rightarrow \infty$; this is

$$
\Phi(y) \sim-\frac{1}{(1-c)^{a}} \frac{\Gamma\left(\alpha+\sqrt{1+\alpha^{2}}\right) \Gamma\left(\alpha-\sqrt{1+\alpha^{2}}\right)}{\Gamma(1+2 \alpha)} \mathrm{e}^{-\alpha y}
$$

with $\Phi$ normalised such that it is unity at the critical layer. We solved for the inviscid incompressible stability problem of the asymptotic boundary layer, using Chebyshev collocation and $N=64$ points. In order to compare to the analytic solution (13) for a given wave frequency $c_{r}$ we first locate the critical layer analytically by

$$
y_{\mathrm{cr}}=-\log \left(1-c_{r}\right) .
$$

While advance knowledge of $y_{\text {cr }}$ could ensure that this point belongs to the spectral grid on which solution of the governing Rayleigh equation is sought, it is impossible (and undesirable from the point of view of the predictive capacity of the numerical approach) to impose this condition at all points where the Rayleigh equation is to be solved numerically for all $c_{r}$. The next step, therefore, is to use a highly accurate interpolation procedure, presently piecewise cubic Hermite, in order to calculate the quantity $\Phi\left(y_{\text {or }}\right) \equiv \Phi_{\text {cr }}$ from our numerically obtained results. Scaling the numerical solution so that $\Phi_{\mathrm{cr}}=1$, we compare a number of eigenvectors resulting from a single calculation against Equation (13). Such a comparison for far-field 
Table 3. Comparison of numerically obtained against analytic solution of the Rayleigh equation for the asymptotic suction boundary-layer profile at $\alpha=0.163$ for calculations at three different grids. Solution corresponding to $c_{r}=0 \cdot 1081$ resolves the critical layer best.

\begin{tabular}{|c|c|c|c|c|c|c|c|c|}
\hline \multicolumn{3}{|c|}{$c_{r}=0.1081$} & \multicolumn{3}{|c|}{$c_{r}=0.0940$} & \multicolumn{3}{|c|}{$c_{r}=0.1033$} \\
\hline$y$ & Numerical & Analytic & $y$ & Numerical & Analytic & $y$ & Numerical & Analytic \\
\hline 3.60 & $4 \cdot 1946$ & 4.2943 & $3 \cdot 91$ & 4.0109 & 4.0743 & $7 \cdot 94$ & $2 \cdot 1171$ & $2 \cdot 1163$ \\
\hline 4.71 & $3 \cdot 5540$ & 3.5815 & 5.15 & $3 \cdot 3132$ & $3 \cdot 3253$ & 9.70 & 1.5879 & 1.5869 \\
\hline $6 \cdot 30$ & $2 \cdot 7620$ & 2.7665 & $6 \cdot 89$ & $2 \cdot 5062$ & 2.5053 & 11.85 & $1 \cdot 1197$ & $1 \cdot 1189$ \\
\hline 8.58 & 1.9066 & 1.9071 & $9 \cdot 32$ & 1.6876 & 1.6858 & $14 \cdot 36$ & 0.7436 & 0.7431 \\
\hline 11.83 & $1 \cdot 1230$ & $1 \cdot 1231$ & $12 \cdot 64$ & 0.9827 & 0.9815 & $17 \cdot 11$ & 0.4751 & 0.4748 \\
\hline $16 \cdot 10$ & 0.5600 & 0.5601 & $16 \cdot 77$ & 0.5016 & 0.5010 & $19 \cdot 75$ & $0 \cdot 3086$ & $0 \cdot 3084$ \\
\hline $20 \cdot 47$ & 0.2744 & $0 \cdot 2744$ & $20 \cdot 74$ & 0.2624 & 0.2621 & 21.75 & 0.2230 & 0.2228 \\
\hline 22.50 & 0.1972 & 0.1972 & $22 \cdot 50$ & 0.1970 & 0.1968 & 22.50 & 0.1973 & 0.1971 \\
\hline
\end{tabular}

values of both the analytic solution and a number of the numerically obtained members of the spectrum is presented in Table 3 . The point made regarding numerical convergence and accuracy now becomes clear, by reference to the different degree of discrepancy between the analytical and the numerical solutions obtained for the different eigenvectors.

While the numerical solution pertinent to all eigenvectors has converged, the results exhibit different degrees of accuracy compared with the (known for this problem) analytical results. The discrepancies were found to stem from the fact that the numerical solutions were obtained on a single grid, the point distribution of which refines the critical layers pertinent to individual eigenvectors to different degrees, a fact which immediately reflects on the accuracy with which the eigenvector is captured.

\subsection{NONLINEAR INVISCID INSTABILITY OF THE COMPRESSIBLE MODEL SHEAR LAYER}

The possibilities of analysis for nonlinear inviscid instability are much more limited as compared to those for the linear problem. Accurate inviscid nonlinear computations are needed in order to aid efforts for both analysis and numerical computations of the viscous problem. In both cases, high-resolution inviscid numerical solutions may identify the critical structures present in the flow field which will require attention in the course of a viscous simulation, while possibly being amenable to analysis. Further, with the issue of the existence of finitetime singularities in the numerical solutions of the inviscid nonlinear stability problem being a subject of current research $[15,16]$, nonlinear inviscid solutions are of interest in their own right. We turn to the presentation of numerical results obtained by application of the algorithms for the direct simulations on the system (2-5). We used the fully spectral algorithm at increasingly high resolutions; for ease of presentation we have first grouped results at four different resolutions, $32^{2}, 64^{2}, 128^{2}$ and $256^{2}$. Given the accuracy properties of the spectral expansions, simulations at typically an order-of-magnitude more points would be needed if the differentiation algorithm were to be based on second-, or fourth-order accurate finite differences. Incidentally, we mention that viscous simulations at modest Reynolds numbers and the relatively low Mach number value used herein, $M=0 \cdot 4$, place much lower computational demands. Sandham and Reynolds [22], using a mixed Fourier-Padé algorithm and the then 
available computing resources, needed a grid of $64 \times 81$ points for their viscous simulations at Reynolds number $\operatorname{Re}=400$. Given the physical background of shear-layer instability and the mathematical nature of the inviscid equations solved, we expect from the outset that any resolution used for the solution of (1) will in the long run become inadequate. Nevertheless, we performed the present simulations with the intention of probing into ever later stages of the nonlinear instability process as the resolution increases. We expect to be able to extract information on which models relevant to the viscous problem may be built.

Of all possible quantities that may be presented we focus on the vorticity; contours at four times, $t=5(5) 20$, may be found in Figure 1. All resolutions may be considered adequate during the early weakly nonlinear stages, confined to a time $t \leqslant 5$; at $t=5$ the low resolution results appear to be marginally deviating from the rest, indicating that they will be of little use for extraction of information as time progresses. At $t=10$ all but the $256^{2}$ results are seen to suffer from a numerical instability originating at the saddle point of this vorticity system. In the $128^{2}$ and more clearly in the $256^{2}$ simulation results it is seen that in the neighbourhood of the saddle point a steep gradient is formed. In a manner analogous to the Burgers equation result presented in the Appendix, poor resolution of this region will result in numerical instability appearing in the full flow field at later times. This is precisely what happens with the three low-resolution simulations at $t \geqslant 15$. Related is the issue of the buildup of steep gradients in the flow. During the weakly nonlinear stages the shape of the vorticity contours is reminiscent of that found in viscous calculations, since the instability we discuss is essentially of an inviscid nature. As time progresses, however, the steepening of the vorticity contours continues unimpeded, given the absence of physical (or numerical) viscosity.

At $t=15$ a number of observations are worthy of discussion. First, the saddle-point region has the hallmarks of a singular region developing in the simulations. This is unlike the corresponding viscous results (and the Burgers equation) where the introduction of even a small amount of viscosity alters the mathematical nature of the system of equations and prevents a singular region from developing. Second, the steep-gradient region marking the outline of the vortex core is substantially less diffuse than its viscous counterpart. This fact alone can account for additional numerical difficulties. Third, one may notice that within the vortex core, a secondary vorticity cluster is being formed, with the tendency to detach itself from the braids. This secondary vorticity pattern can be inferred already from the $t=10$ results and is clearly seen in the $256^{2}$ results at $t=20$, when a replicated copy of the original vorticity pattern is encompassed by the braids. At the same time, the $256^{2}$ calculation is seen to suffer from generation of numerical instability at the saddle point region. Consequently, we may only draw qualitative conclusions from the high-resolution results at $t=20$, when using up to $256^{2}$ points.

We return to the question which gave rise to the nonlinear results of the present paper. Already at $t=15$ it is easy to identify in both the $64^{2}$ and the $128^{2}$ simulation results clusters of vorticity trapped in between the steep-shear gradients, analogous to those which gave rise to the question posed at the outset of the present investigation [24]. Incidentally we mention that the simulations in [24] were performed at analogous resolutions, albeit based on (1). Although they are certainly not pointwise oscillations, these vorticity clusters become narrower as resolution increases and (almost) vanish in the result of the $256^{2}$ calculation. The conclusion to which we are led is that, at least within the context of the present inviscid simulations, these vorticity regions are not the result of some physical, but rather a numerical instability. 

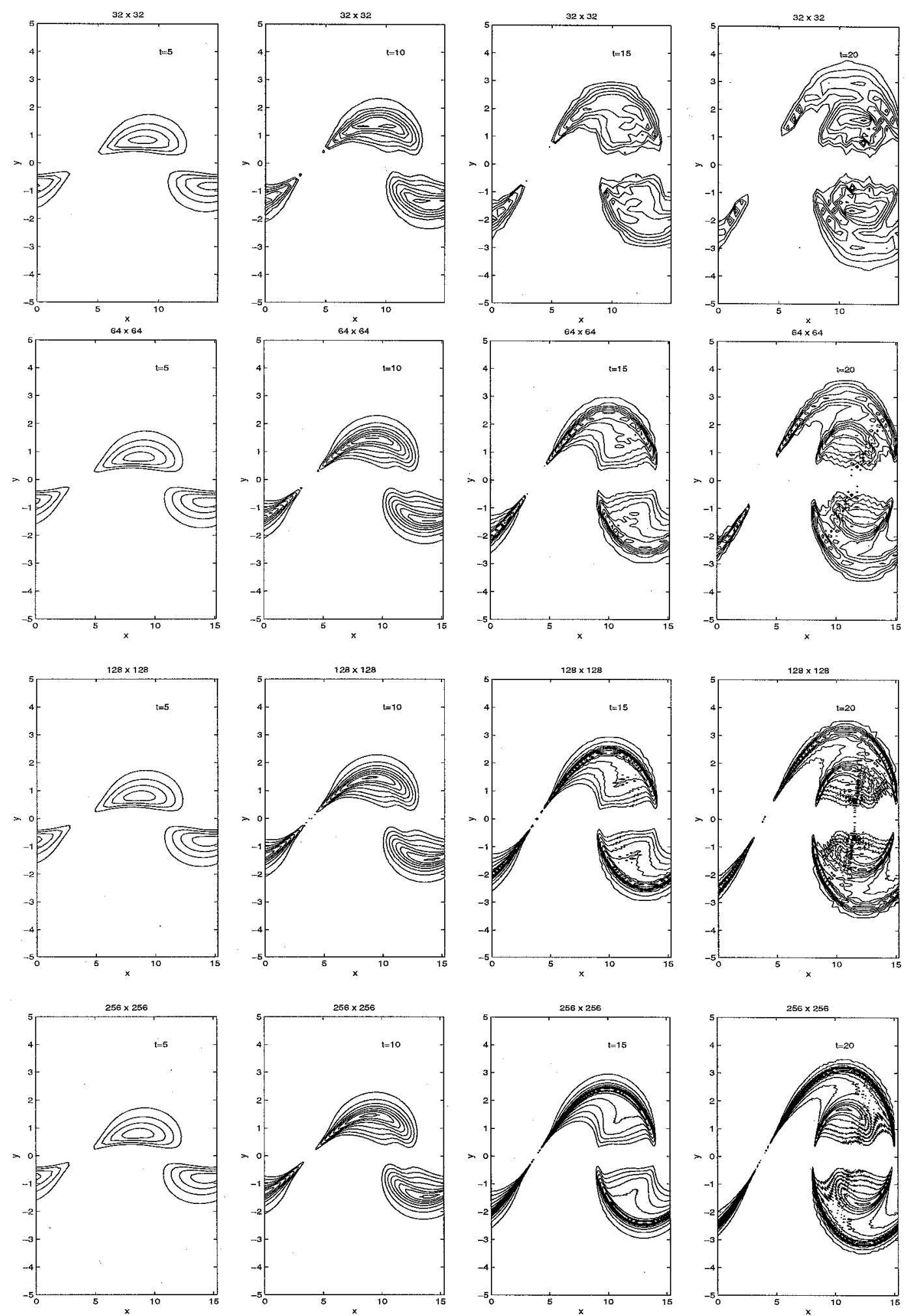

Figure 1 . 


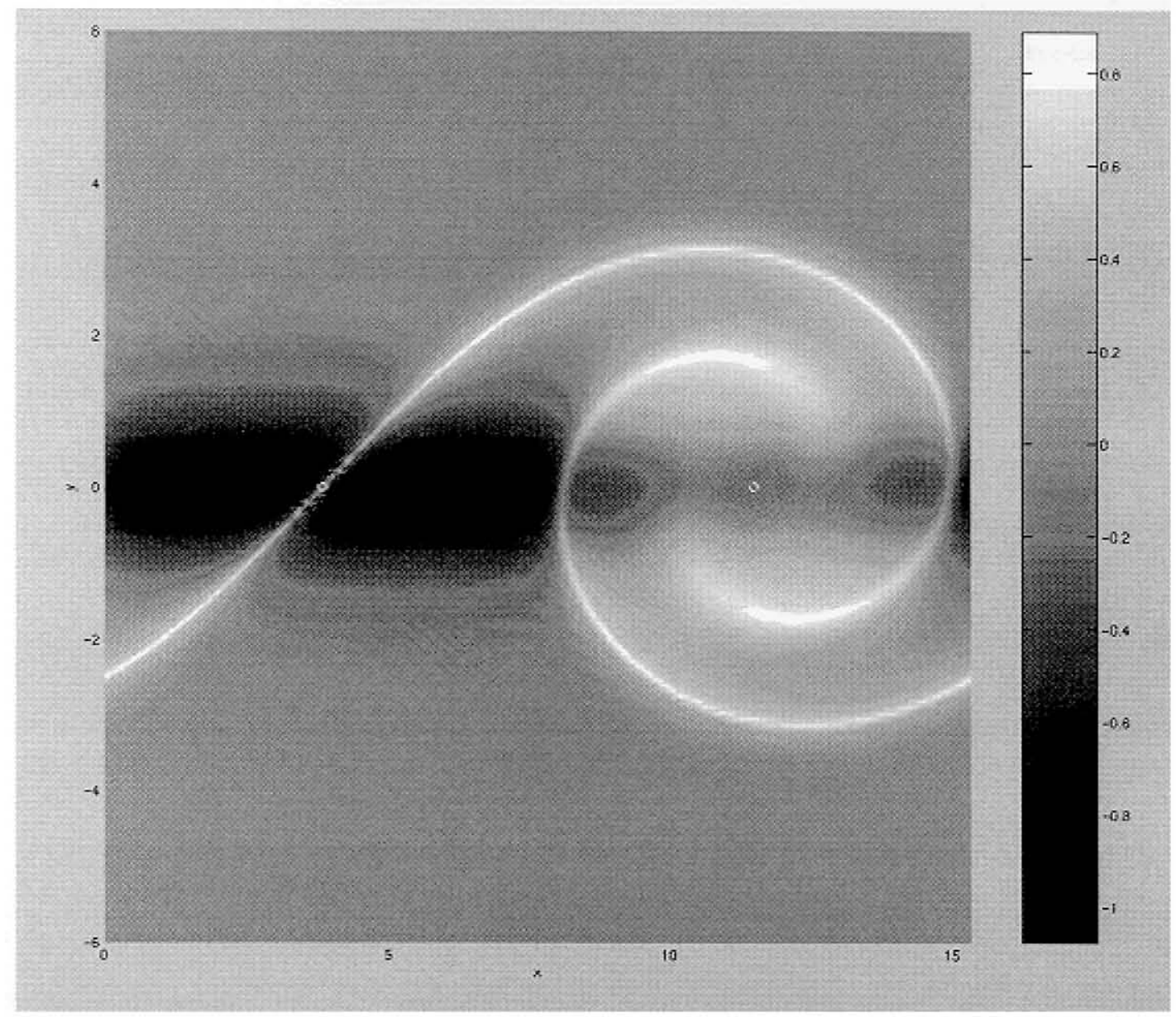

Figure 2.

At $t=20$ the situation, as far as the saddle point and the outline of the vortex core is concerned, remains unaltered. The secondary structure known from viscous simulations and its symmetric structure (with respect to the vortex-core centre) are seen to develop. The structure at $y>0$ might be thought of as being related to the tail of the vortex-core perimeter at $y<0$ and vice versa, as seen in Figure 2, where the narrowness of the peak vorticity regions may be appreciated. Topological analysis of the flowfield [32] reveals further critical points, besides the saddle point (SP) and the vortex core (VC). These are identified with reference to their location from left to right on the centreline as points $\mathrm{P} 1$ and $\mathrm{P} 2$, the first lying at a small distance to the right of $\pi / \alpha$, between the tip of the lower braid and that of the secondary vorticity cluster formed in the upper half of the domain. Point P2, on the other hand, is located by the same amount to the left of $2 \pi / \alpha$ and may be defined accordingly as the location between the the forward tip of the upper braid and that of the secondary vorticity in the lower half of the domain.

As far as the region in the neighbourhood of the saddle point is concerned, we observe that no appreciable difference in the level of pressure exists, indicating that no shock formation should be expected there. By contrast, the difference in the level of vorticity at either side of either P1 or P2 is appreciable. This suggests that at P1 and P2 shocks may develop as 
the Mach number increases; this would require additional attention to be paid in adequately resolving the neighbourhood of these points if the simulation is to progress to higher Mach numbers and the equations are to be integrated for longer times. Inside the vortex core, on the other hand, one may identify points which subdivide the vortex core in three regions, their role being analogous to that of $\mathrm{P} 1$ and $\mathrm{P} 2$ at earlier times; extrapolating from the present results, we expect the formation of tertiary structures within the vortex core. For such a claim to be substantiated, however, simulations at even higher resolutions are necessary.

Such simulations have been performed for $512^{2}$ spectral collocation points. The results, again in the form of vorticity contours, are presented at times $t=10(5) 35$ in Figure 3. Of interest in the results of these numerically nontrivial simulations are a number of findings. First, the suggestion that was put forward by reference to the lower resolution $256^{2}$ simulations regarding shock formation is erroneous. The uneven pressure distribution at either side of points $\mathrm{P} 1$ and $\mathrm{P} 4$ at $t=20$ is absent at later times. Instead, these points are simply found to subdivide what initially used to be the vortex core into smaller regions inside which the original pattern of vorticity has replicated itself. Although, eventually, numerical instability will destroy these simulations also (as it would destroy simulations at any higher resolution), the qualitative picture obtained is the following.

At time $t \leqslant 10$ a secondary cluster of vorticity is being formed and detaches from the braids. The latter, unimpeded by the absence of viscosity, steepen significantly more than their respective viscous counterparts, as seen in the $t=15$ results. The secondary vorticity generated initially in the neighbourhood of the tip of the lower braid, extends over an appreciable portion of the vortex core by $t=20$. At that time, the flow may be seen as being composed of the steep gradient region outlining the core of the vortex and the core itself which contains a pattern of vorticity qualitatively analogous to that imposed originally. At $t \geqslant 20$ a repetition of the process discussed up to $t=20$ takes place within the vortex-core region. On the one hand, the original braids continue steepening, while inside the vortex core a secondary high-shear region develops, at the same time shedding a tertiary cluster of vorticity of smaller scale. The secondary high-shear region steepens as time progresses; the tertiary vortex system sheds a smaller-scale copy of itself further inwards towards the vortex core and this cascade continues, for as long as qualitatively results may be extracted from the present simulations. Four regions of high shear can be identified from the numerical instability in the $512^{2}$ simulation results of Figure 3 at $t=35$. Shortly after this time this numerical instability, resulting for the reasons discussed, leads to numerical breakdown of the simulation.

Based on the results obtained we conjecture that, in the course of a high-Reynolds-number viscous simulation, the succession of high-shear regions generated within the vortex core as time progresses is one of the critical flow locations which places high resolution demands. The nontrivial shape of the steep gradients, though, may result in the distribution of points devoted to capturing the phenomenon being nonstandard. Adaptive grid generation, within the frame of spectral-element computations appears to be one of the ways forward to address this numerically challenging problem

\section{Discussion}

The maturing of numerical algorithms and computing hardware to support direct numerical simulations results in a tendency to rely on numerical solutions alone in order to describe the complex phenomena associated with transition of fluid flow from a laminar to a turbulent state. 

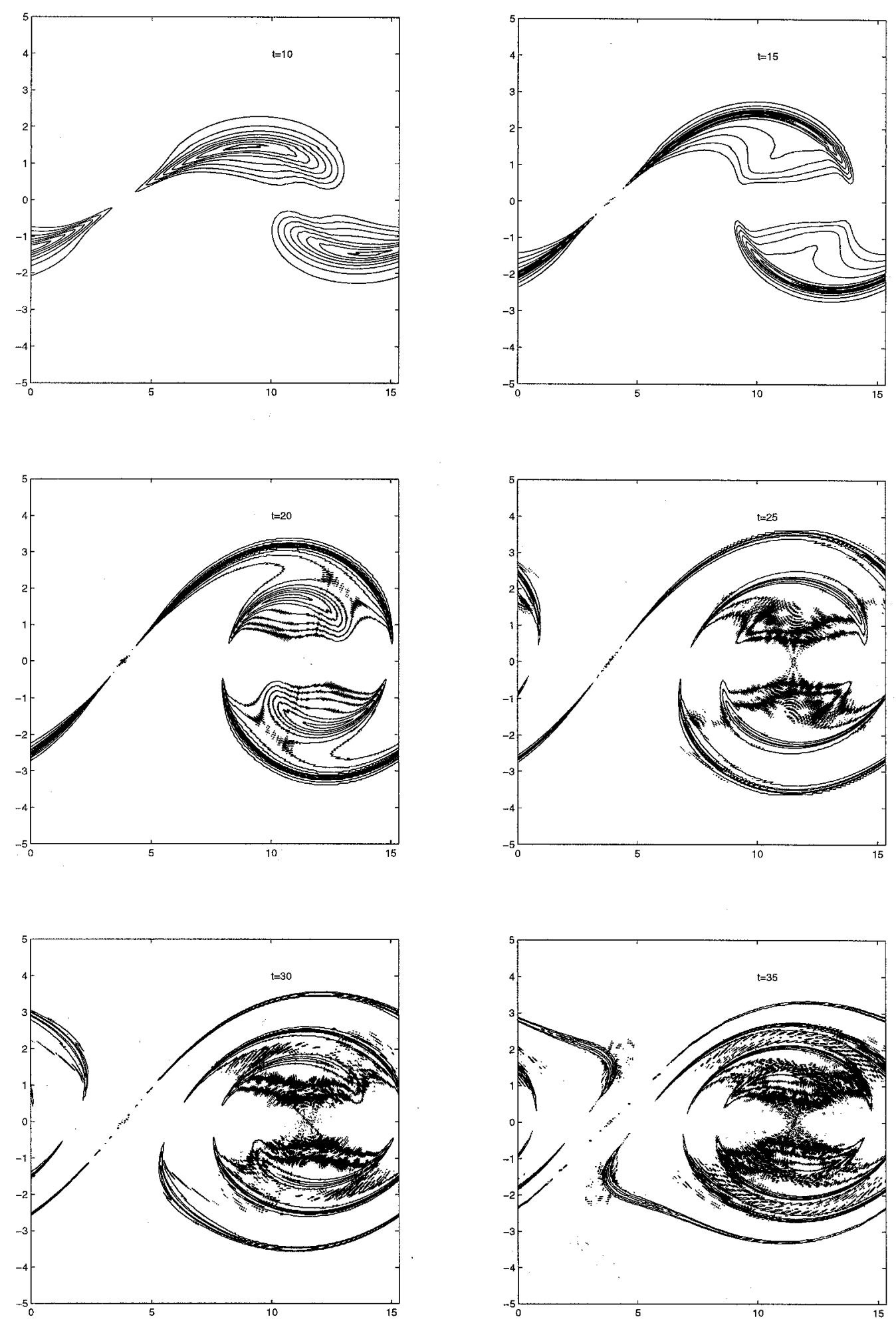

Figure 3. 
This approach is fully acceptable when used critically and has, indeed, resulted in spectacular successes. The danger of relying exclusively on the numerics, however, is inherent in the nature of the system of equations solved. Nonlinearity has the potential of delivering unphysical solutions, even in the presence of convergence, simply on account of the use of a single grid which cannot describe all instability modes present in the flow with the same degree of accuracy. If the inaccurately described modes become physically relevant, for instance through some receptivity mechanism [34], numerically correct but physically irrelevant solutions may be obtained.

We have briefly discussed the inviscid linear problem and presented the asymptotic suction boundary-layer model flow, in which converged numerical solutions of the inviscid linearstability eigenvalue problem may be derived which exhibit different degrees of relevance to the physics of the instability problem. For accurate (linear) stability results, resolution of the critical layer is shown to be the condition to be satisfied, additionally to that of basic flow gradient resolution. Unlike the case of the shear layer, where the condition of coincidence of linear critical layer and location of maximum basic flow derivatives is fortuitously satisfied, this result serves to illustrate the fact that a single grid cannot represent all instability modes present in the flow to the same degree of accuracy, if critical layers exist in regions other than that of maximum basic flow derivatives. Such regions can be generated in the course of nonlinear time-dependent simulations. The implications for modest-resolution nonlinear computations based on low-order numerical methods are obvious.

Next, we turned our attention to the nonlinear inviscid instability of the compressible two-dimensional model shear-layer flow. We addressed the issue of efficiency, which is paramount to the success of high-resolution simulations for which long-time integration has to be performed. The optimum approach turns out to be dependent on the machine used and the quality of optimisation of the software used for the residual calculations. High-resolution spectrally accurate nonlinear instability results have been obtained, motivated by a question which arose in the course of both viscous and inviscid direct simulations of this classic flow. The localised regular pattern of vorticity concentrations seen in the early simulations is, at least in the inviscid framework considered, found to be numerical in origin, related to the saddle-point at $x=\pi / 2 \alpha$. The development of steep gradients in the flow is documented. The nonlinear instability of the inviscid model is found to comprise all the characteristics known from viscous simulations, albeit that the flow gradients are much steeper and can lead, in this inviscid problem, to numerical difficulties not experienced in direct simulations in the presence of viscosity. On the other hand, precisely because of this reason, it is much easier to identify the flow regions which become critical in the course of the simulations and will need particular attention in order for either viscous or inviscid simulations to proceed in time, while delivering physically meaningful results. The present inviscid nonlinear stability calculations are a step in this direction and demonstrate that the model problem discussed will continue to be both a theoretical and a numerical challenge in the foreseeable future. In the meantime, the present results serve to underline what is, hopefully, obvious to the community of numerical modellers, namely that computation should be accompanied and, whenever possible, preceded by analysis. 
Table 4. Convergence history of the slope $\partial \bar{u} / \partial x(x=0)$ in the numerical solution of Burgers's equation (14).

\begin{tabular}{rlll}
\hline$N x$ & $\Delta t$ & $t_{0}$ & $\partial \bar{u} / \partial x(x=0)$ \\
\hline 32 & $5.0(-4)$ & 0.51250 & 151.4240 \\
64 & $5 \cdot 0(-4)$ & 0.51050 & 152.0031 \\
128 & $2 \cdot 5(-4)$ & 0.51050 & 152.0046 \\
256 & $7 \cdot 5(-5)$ & 0.51075 & 152.0049 \\
\hline
\end{tabular}
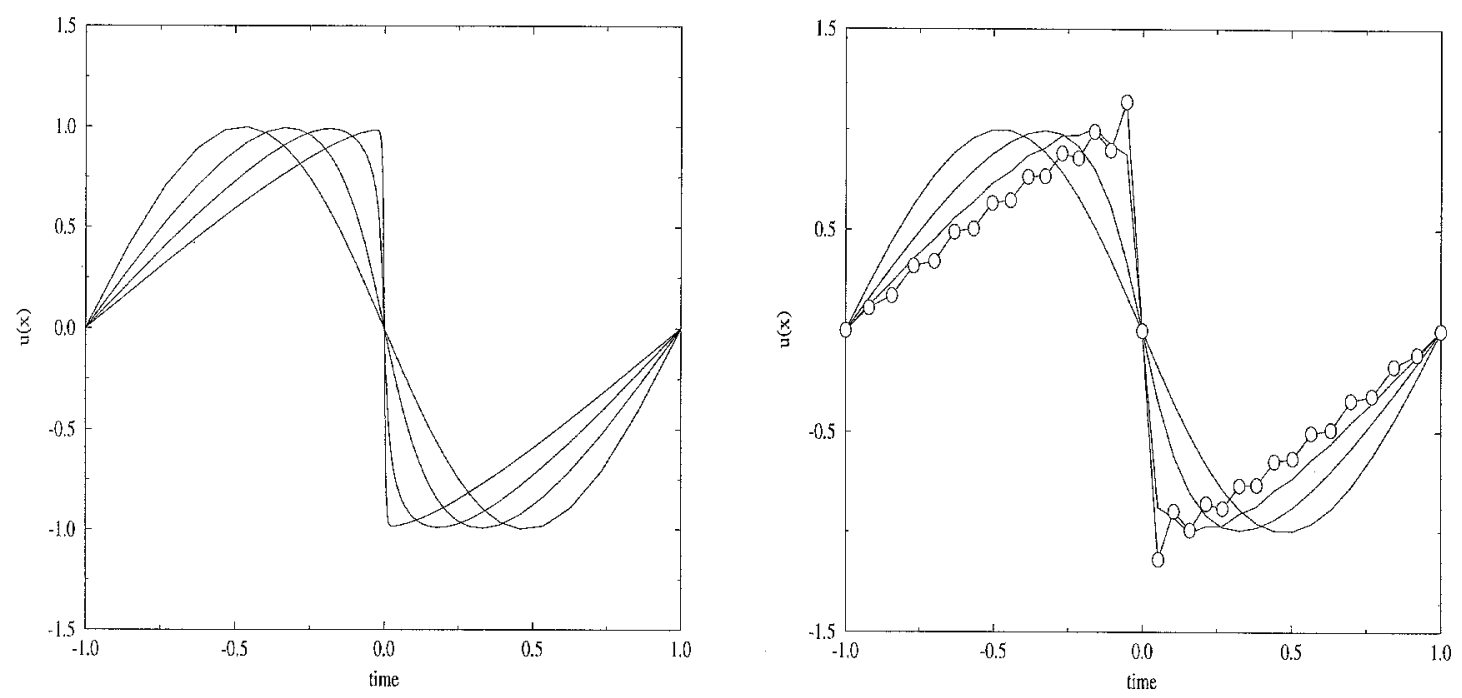

Figure 4 .

\section{Appendix. Spectral methods and finite gradients}

The issue of resolution of sharp gradients by a spectral method is introduced clearly by reference to numerical solutions of the one-dimensional Burgers equation,

$$
\frac{\partial \bar{u}}{\partial t}+\bar{u} \frac{\partial \bar{u}}{\partial x}-v \frac{\partial^{2} \bar{u}}{\partial x^{2}}=0
$$

in $x \in[-1,1]$. We solve (A1) subject to the initial condition $u(x, t=0)=-\sin (\pi x)$ and the boundary conditions $u(x=-1, t)=u(x=1, t)=0$, using Fourier collocation and the mixed implicit/explicit scheme due to Spalart et al. [25] for the time-stepping of the viscous and nonlinear term, respectively. The viscosity parameter in this example was chosen $v=0.01 / \pi$ at which this model equation develops a steep gradient at time $t_{0} \approx 0.5$. The performance of the collocation scheme used for the direct simulations is tested against the analytically known result for the maximum value of the slope of $\partial \bar{u} / \partial x(x=0)=$ 152.0052 [26]. Table 4 presents grid-sequencing results of single-domain Fourier collocation; the number of Fourier collocation points $N x$ is shown; the time step is $\Delta t$ and the time at which the maximum slope occurs is $t_{0}$. No cosmetic postprocessing of the results has been 
performed and values at the cited times are presented without any attempt to obtain the maximum value of the derivative through some interpolation procedure.

What is of significance here is that, although the convergence towards the analytic value is evident, in order for results of such quality to be obtained the neighbourhood of $x=0$ must be well resolved; the fact that this region becomes interesting in the course of the simulation is, of course, known a-priori in this simple flow model. An inadequate resolution of the region where the gradient develops, in conjunction with the global nature of the spectral method for the calculation of spatial derivatives, results in the whole flow field being contaminated by numerical instability. This is clearly demonstrated in Figure 4 where both the well resolved results, as well as those obtained by naive use of the equidistantly distributed Fourier collocation points are presented.

Unlike a low-order accurate finite-difference calculation, in which the localised pointwise oscillations might be considered acceptable, the global contamination of the flowfield obtained from a spectral method leaves little room for misinterpretation of inaccurate numerical results as potentially physical. Such situations of localised in space steep flow gradients are common during the late transitional stages of either wall-bounded or free-shear flows. Aside from guidance received from linear and nonlinear inviscid analysis (cf. [3, 4, 9], and [10]), highresolution inviscid nonlinear results, such as those obtained herein, are necessary to highlight the physical mechanisms involved prior to a (substantially more expensive) three-dimensional simulation of the viscous problem.

\section{Acknowledgements}

It is a pleasure to contribute the present paper on the occasion of Professor P. J. Zandbergen's retirement and concurrent assumption of the duty of Chairman of the Royal Netherlands Academy of Arts and Science. The present work commenced at the University of Twente in the group of Professor Zandbergen at a time when freedom, resulting in uninhibited creative thought, was still an affordable commodity within Academia.

The author gratefully acknowledges support granted by an Alexander von Humboldt Research Fellowship to pursue the present work at the Division of Transition and Turbulence of the DLR in Göttingen. Discussions with Dr. U. Dallmann on the issue of identification of flow structures are kindly appreciated.

\section{References}

1. L. Kleiser and T. A. Zang, Numerical simulation of transition in wall-bounded shear flows. Ann. Rev. Fluid Mech. 23 (1991) 495-537.

2. L. M. Mack, Boundary layer stability theory. Doc. 900-277 Jet Propulsion Laboratory, Pasadena, CA., (1969) $1.1-15.7$ ( 2 vols. $)$.

3. P. W. Duck, The inviscid axisymmetric stability of the supersonic flow along a circular cylinder. NASA CR-181816 and J. Fluid Mech. 214 (1990) 611-637.

4. C. C. Lin, The Theory of Hydrodynamic Stability. Cambridge: Cambridge University Press (1955) $155 \mathrm{pp}$.

5. L. M. Mack, Boundary layer linear stability theory. AGARD Rep.709 (1984) 3.1-3.81.

6. M. G. Macaraeg and C. L. Streett, New instability modes for bounded free stream flows. Phys. Fluids A 1 (1989) 1305-1307.

7. S. J. Shaw and P. W. Duck, The inviscid stability of supersonic flow past heated or cooled axisymmetric bodies. Phys. Fluids A4 (1992) 1541-1557.

8. F. T. Smith and O. R. Burggraf, On the development of large-sized short-scaled disturbances in boundary layers. Proc. R. Soc. London A399 (1985) 25-55. 
9. Yu.-S. Kachanov O. Ryzhov and F. T. Smith, Formation of solitons in transitional boundary layers: theory and experiment. J. Fluid Mech. 251 (1993) 273-297.

10. O. S. Ryzhov, The development of nonlinear oscillations in a boundary layer and the onset of random disturbances. Rensselaer Polytechnic Institute Math. Report 213(1994) 14pp.

11. G. Erlebacher and M. Y. Hussaini, Numerical Experiments in supersonic boundary layer stability. Phys. Fluids A 2 (1990) 94-104.

12. W. Koch, On a degeneracy of temporal secondary instability modes in Blasius boundary-layer flow. J. Fluid Mech. 243 (1992) 319-351

13. R. Fjørtøft, Application of integral theorems in deriving criteria of instability for laminar flows and for the baroclinic circular vortex. Geofys. Publ. 17 (1950) 52pp.

14. J. T. Stuart, Nonlinear Euler partial differential equations: singularities in their solution. In: D. J. Benney, F. H. Shu and C. Yuan (eds.) Symposium to honour C. C. Lin. World Scientific (1987) 81-95.

15. R. M. Kerr, Evidence for a singularity in the three-dimensional incompressible Euler equation. Phys. Fluids A5(1993) 1725-1746.

16. S. Tanveer, A note on singularities of the 3D Euler equation. ICASE Rep. 94-93 (1994) 12pp.

17. P. Hall and N. J. Horseman, The linear inviscid secondary instability of longitudinal vortex structures in boundary layers. J. Fluid Mech. 232 (1991) 357-375.

18. A. W. Vreman, B. J. Geurts, and J. G. M. Kuerten, Shocks in direct numerical simulation of the confined three-dimensional mixing layer. Phys. Fluids 7 (1995) 2105-2107.

19. S. Lele, Compact finite-difference schemes with spectral-like resolution. J. Comp. Phys. 103 (1992) 16-42.

20. N. A. Adams, An explicit temporal spectral/finite-difference method for the direct simulation of compressible boundary layer transition. Deutsches Zentrum für Luft und Raumfahrt DLR IB 221-92 A 25(1992) 76pp.

21. H.-C. Ku, and D. Hadziavramidis, Chebyshev expansion methods for the solution of the extended Graetz problem. J. Comp. Phys. 56 (1984) 495-512.

22. N. D. Sandham, and W. C. Reynolds, A numerical investigation of the compressible mixing layer. Report No. TF-45 Dept. of Mechanical Engineering, Stanford University (1989) 244pp.

23. A. A. Wray, Very low storage time-advancements schemes. NASA Ames Internal Report (1986) 6pp.

24. V. Theofilis, On secondary destabilisation of an attachment-line boundary layer in compressible flow. In: R.A.W.M. Henkes and J.L. van Ingen (eds.) Transitional Boundary Layers in Aeronautics Colloquium of the Royal Netherlands Academy of Arts and Sciences. North-Holland (1996) 199-206.

25. P. R. Spalart, R. D. Moser, and M. M. Rogers, Spectral methods for the Navier-Stokes equations with one infinite and two periodic directions. J. Comp. Phys. 96 (1991) 297-324.

26. M. G. Macaraeg, and C. L. Streett, Improvements in spectral collocation discretisation through a multiple domain technique. Appl. Num. Math. 2 (1986) 95-108.

27. L. Hocking, Nonlinear instability of the asymptotic suction velocity profile. Q. J. Mech. Appl. Math. 28 (1975) 341-353.

28. P. Hall M. R. Malik and D. I. A. Poll, On the stability of an infinite swept attachment-line boundary layer. Proc. Roy. Soc. London A 395 (1984) 229-245.

29. V. Theofilis, On linear and nonlinear instability of the incompressible swept attachment line boundary layer. J. Fluid Mech. 355 (1998) 193-227.

30. T. H. Hughes and W. H. Reid, On the stability of the asymptotic suction boundary layer profile. J. Fluid Mech. 23 (1965) 715-735.

31. C. Canuto M. Y. Hussaini A. Quarteroni and T. A.Zang, Spectral Methods in Fluid Dynamics, Berlin: Springer Verlag (1993) 557pp.

32. U. Dallmann, H. Vollmers and W-H Su, Flow topology and tomography for vortex identification in unsteady and in three-dimensional flows. In: J.N. Sørensen (ed.) Proceedings of IUTAM Symposium Simulation and Identification of Organized Structures in Flows, Techn. Univ. Denmark, Lyngby Kluwer (1997) 16pp.

33. J. P. Boyd, Chebyshev and Fourier Spectral Methods. Lecture Notes in Engineering 49 Springer (1989) 792pp.

34. F. P. Bertolotti, and U. Dallmann, New models for the response of two-dimensional boundary layers to forcing by free-stream vorticity and for the response of three-dimensional boundary layers to forcing by localised surface irregularities. Euromech Colloquium 359 Stability and Transition of Boundary-Layer Flows. Stuttgart 1997 (unpublished) 